\title{
Spectroscopic Ellipsometry of Mucin Layers on an Amphiphilic Diblock Copolymer Surface
}

\author{
NATALIYA A. NIKONENKO, ${ }^{\mathrm{a}, *}$ IBRAHEEM A. BUSHNAK, \\ and JOSEPH L. KEDDIE ${ }^{\mathrm{b}}$ \\ a B.I. Stepanov Institute of Physics, National Academy of Sciences of Belarus, \\ Nezalezhnasti Ave. 68, 220072 Minsk, Belarus \\ ${ }^{\mathrm{b}}$ Department of Physics, University of Surrey, Guildford, Surrey GU2 7XH, UK
}

\section{ABSTRACT}

Both visible and infrared (IR) spectroscopic ellipsometry have been employed to study the structure of thin layers of bovine submaxillary mucin (BSM) adsorbed on poly(acrylic acidblock-methyl methacrylate) (PAA- $b$-PMMA) copolymer and poly(methyl methacrylate) surfaces at three $\mathrm{pH}$ values $(3,7$ and 10). The adsorbed mucin layer on the copolymer surface had the greatest thickness $(17 \mathrm{~nm})$ when adsorbed from a mucin solution at a $\mathrm{pH}$ of 3 . For the first time, IR ellipsometry was used to identify adhesive interactions and conformational changes in mucin/polymer double layers. After applying the regularized method of deconvolution in the analysis, the formation of hydrogen bonds between the carboxyl groups of the BSM and PAA- $b$-PMMA copolymer in double layers has been found. The IR ellipsometry data, in agreement with the visible ellipsometry analysis, indicate the $\mathrm{pH}$ dependence of adhesion of mucin to the copolymer surface. There is an increase in the amount of hydrogenbonded carboxyl groups in mucin deposited at a pH of 3. There is no evidence that the mucin's amide groups participate in this bonding. At the lower $\mathrm{pH}$, the IR ellipsometry spectra after deconvolution reveal an increase in the proportion of $\beta$-sheets in the BSM upon adsorption on the copolymer surface, indicating a more unfolded, aggregated structure. The IR ellipsometry data also indicated some changes in the conformational states of the side groups in the copolymer induced by entanglements and bonding interactions with the mucin macromolecules. Deconvolution provides an unprecedented level of information from the IR ellipsometry spectra and yields important insights.

Index Headings: IR ellipsometry; IR spectroscopy; Deconvolution; Mucoadhesion; Hydrogen bonding; Mucin 
Mucins are natural amphiphilic biomolecules that have potential applications in biotechnology. ${ }^{1-4}$ It has been shown recently ${ }^{5,6}$ that mucin layers adsorbed on polymer surfaces reduce bacterial adhesion. Hence, creating robust mucin coatings and exploring their structure and properties is an important step in the development of improved coatings.

The mucin macromolecule is considered to be a block copolymer made up of a long, linear peptide backbone ( $20-50 \%)$ with regions of densely-packed linear or branched carbohydrate side chains $(\sim 50-80 \%)$ and "bare" regions with no or few carbohydrate side chains. ${ }^{1-3}$ Molecular weights range from 0.5 to $40 \times 10^{6}$ Daltons, depending on the source. ${ }^{1,2}$ The carbohydrate branched chains of mucin have a large charge density mainly because of sialic acid and sulfate residues. Mucin has an overall negative charge in neutral solutions, which diminishes as the $\mathrm{pH}$ decreases. ${ }^{3}$ It is worth noting that the composition, structure, conformation and interfacial properties of mucins differ somewhat depending on their origin. ${ }^{1,2}$

Mucin adheres to hydrophobic surfaces via its unglycosylated, hydrophobic protein blocks, while glycosylated hydrophilic blocks tend to stretch out away from the surface. ${ }^{5}$ Hence, adsorbed mucin layers impart hydrophilicity to an otherwise hydrophobic surface. It has been reported earlier that mucin coatings on hydrophobic polymeric materials, such as poly(methyl methacrylate) (PMMA) and polystyrene, can suppress the adhesion of some bacterial species due to the reduction of the hydrophobicity of their surface. ${ }^{5,6}$ However in these previous works, ${ }^{5,6}$ there was no attempt to control the adsorbed mucin layer thickness or to explore the structure of mucin layers adsorbed onto polymer surfaces.

Our approach to creating the mucin coatings has been inspired by previous studies of mucoadhesion, a term which refers to the attachment of materials to a mucus layer. ${ }^{2-4}$ It has been well documented that there is strong adhesion between mucin macromolecules and poly(acrylic acid) (PAA) in aqueous solutions. ${ }^{3,7-10} \mathrm{We}$ therefore propose to use an amphiphilic diblock copolymer of PAA and PMMA (PAA- $b$-PMMA) with the aim of attaching mucin to the polymer surface through mucoadhesive interactions. Whereas previous studies of bacterial reduction relied on mucin adsorption through hydrophobic interactions ${ }^{5,6}$ the present approach relies on hydrogen bonding, as will be demonstrated herein.

The purpose of this work is to explore the structure of bovine submaxillary mucin (BSM) layers adsorbed on PAA- $b$-PMMA copolymer surfaces and to identify the molecular mechanisms of the adhesion and conformational changes in the BSM/PAA-b-PMMA doublelayer system using spectroscopic ellipsometry. In this work we also determine the effect of $\mathrm{pH}$ 
to more hydrophobic PMMA.

70 The exact mechanisms of mucoadhesion are under continuing investigation. ${ }^{2-4}$ In the 71 literature, at least three mechanisms for mucin/PAA adhesion in aqueous solutions have been 72 proposed. One mechanism involves electrostatic interaction and hydrogen bonding mainly 73 between the carboxyl and sulphate groups in the mucin's carbohydrate side chains and the 74 carboxyl groups in poly(acrylic acid). ${ }^{3,7-9}$ In aqueous solutions, the hydrophobic protein 75 backbone of mucin is largely protected by the carbohydrate side chains, which are exposed to 76 the water due to their hydrophilic nature. ${ }^{3}$ A second possible mechanism, proposed by Patel et 77 al., ${ }^{10}$ is that amide groups of the mucin participate in hydrogen bonding with the carboxyl 78 groups of PAA. Furthermore, interpenetration of mucin into polymers and the creation of 79 molecular entanglements provide a physical adhesion mechanism. ${ }^{3,4,7-9}$ This third mechanism is 80 dependent on the swelling behaviour of the mucin and the polymer, and it is time-dependent.

81 Various spectroscopic methods, in particular attenuated total reflection Fourier transform 82 infrared (ATR-FTIR) spectroscopy, FTIR and nuclear magnetic resonance spectroscopy, 83 dynamic light scattering, together with atomic force microscopy have been successfully applied 84 to study the complex structure of mucin and molecular mechanisms of its adhesion. ${ }^{8-13}$ 85 Considerable attention has been given to understanding the adsorption behavior of mucin and 86 its important functional properties. ${ }^{14-17}$ However, the molecular structure of mucin layers 87 adsorbed onto polymer surfaces has not been sufficiently explored so far. Little is also known 88 about the conformational changes of mucin that might occur, as is the case for proteins, upon adsorption onto various polymer surfaces. ${ }^{16-18}$

90 Over the past decade, IR ellipsometry has been demonstrated to be very sensitive 91 technique for structural analysis of thin films and multi-layers. ${ }^{19-21}$ Ellipsometry in the infrared 92 range shows also considerable promise as a tool for exploring the molecular structure of thin 93 biological layers, and hence is well suited to studies of mucoadhesion in multi-layered systems.

94 This non-invasive technique can study thin multi-layers with much greater sensitivity than can 95 be obtained from transmission spectroscopy, ${ }^{22}$ but with greater penetration depths than 96 afforded by internal reflection spectroscopy yielding information on thickness. ${ }^{19}$ It has been 97 applied with good success to studies of interfacial chemistry ${ }^{23}$ and bonding, ${ }^{24,25}$ to polymer 98 orientation, ${ }^{26}$ to interdiffusion in polymer bilayers ${ }^{27,28}$ and polymer brushes. ${ }^{29}$ There are a few 99 examples of applications of IR ellipsometry to the study of biological molecules in thin films 100 and at interfaces, ${ }^{19,30-32}$ but - until now - the technique has not been applied to detailed studies 101 of biomolecular interfacial bonding and conformation.

102 In our work we apply the IR ellipsometry technique to identify structural and 
conformational changes in BSM/PAA- $b$-PMMA double layers caused by mucin adhesion to a

104 copolymer surface. Whereas there is previous study of mucin/PAA adhesion in aqueous

105 solutions, ${ }^{2-4,7-10}$ we are not aware of any reported investigations of mucin adsorption on an

106 amphiphilic copolymer, such as PAA-b-PMMA. We also use spectroscopic ellipsometry in the

107 visible range to examine the thicknesses of adsorbed mucin layers on the PAA- $b$-PMMA

108 copolymer, as compared to PMMA surface. The application of regularized method of

109 deconvolution ${ }^{33,34}$ to the IR ellipsometry spectra allows us to reveal the fine structure of the

110 complex bands and greatly increases the amount of useful information obtained via the IR

111 ellipsometry analysis.

\section{MATERIALS AND METHODS}

114 Materials. PAA- $b$-PMMA was used as received from Polymer Source, Inc. (Montreal, 115 Canada). The molecular weights of the blocks were $2.8 \times 10^{4} \mathrm{~g} / \mathrm{mole}$ for the PAA block and 1.0 $116 \times 10^{4} \mathrm{~g} /$ mole for the PMMA block. The molecular weight polydispersity was $\mathrm{M}_{\mathrm{w}} / \mathrm{M}_{\mathrm{n}}=1.14$. 117 Poly(methyl methacrylate) was purchased from Polymer Laboratories, UK $\left(\mathrm{M}_{\mathrm{w}}=8.0 \times 10^{4}\right.$ $118 \mathrm{~g} / \mathrm{mole}$ ). Bovine submaxillary mucin, type 1-S as-lyophilized powder with approximately $12 \%$ 119 bound sialic acid (Sigma Inc.), was used as received.

120 The experiments used two different types of substrates: (1) silicon and (2) gold. Single121 crystal (100) wafers were used as-received for all visible ellipsometry experiments. A typical 122 thickness of the native $\mathrm{SiO}_{2}$ layer was determined to be $2.5 \mathrm{~nm}$. Gold substrates, used for all 123 infrared ellipsometry analysis, were prepared by the thermal evaporation of gold onto cleaned 124 glass slides to create $55 \mathrm{~nm}$ films. Gold and silicon substrates were used because of their high 125 IR and visible light reflectivity, respectively.

126 Thin Film Preparation. Copolymer films (ca. $150 \mathrm{~nm}$ thick) were created by spin-casting 127 PAA-b-PMMA 3 wt.\% solutions in tetrahydrofuran onto the gold substrates using a photoresist 128 spin-coater (Cammax Precima Ltd., Colchester, UK) at $2000 \mathrm{rpm}$ for $6 \mathrm{~s}$. Thinner copolymer 129 films (18-20 nm) were spin-cast onto the silicon substrates from $0.5 \mathrm{wt} \% \%$ solutions. PMMA 130 films were spin-cast from toluene solutions. All types of polymer films were heated in a 131 vacuum at $90^{\circ} \mathrm{C}$ for $1 \mathrm{~h}$ to remove remanent solvent and to allow the polymer conformation to 132 relax.

133 In the IR ellipsometry analysis, single films (copolymer or mucin) were first examined to 134 identify the characteristic bands. Bilayers consisting of a mucin film on a copolymer film on a 135 gold substrate were also examined. Mucin films were deposited from $1 \mathrm{mg} / \mathrm{ml}$ solutions of 136 BSM in de-ionized (DI) and filtered water. Approximately $150 \mu 1$ of the solution was spread 
137 across an area of $3 \mathrm{~cm} \times 4 \mathrm{~cm}$, and the film was left to dry in a desiccator containing silica gel

138 for $24 \mathrm{~h}$. The resulting mucin film thicknesses ranged between 400 and $500 \mathrm{~nm}$. Mucin

139 solutions were made at one of two $\mathrm{pH}$ values ( 3 and 7) obtained by the addition of a $0.1 \mathrm{M} \mathrm{HCl}$

140 solution or a $1 \mathrm{M} \mathrm{NaOH}$ solution. BSM/PAA-b-PMMA bilayers were created by successive

141 deposition of the copolymer followed by the mucin films on gold substrates by the procedures 142 just described.

143 Very thin mucin layers were also created by adsorption from mucin solutions onto one of 144 three different surfaces: PMMA; PAA- $b$-PMMA; and silicon (for comparison). The substrates 145 were soaked in $1 \mathrm{mg} / \mathrm{ml} \mathrm{BSM}$ solution for $24 \mathrm{~h}$ while shaking. The $\mathrm{pH}$ values of the BSM 146 solutions were adjusted to 3, 7 or 10 . The substrates were then removed and rinsed three times 147 with DI water to remove any unattached mucin from the surface. The films were left to dry in a 148 desiccator at $\sim 0 \%$ RH. These adsorbed mucin films were then analysed with visible 149 ellipsometry technique. The polymers and mucin are both glassy, and neither is subject to 150 crystallization. They can be considered to be isotropic.

151 Spectroscopic Ellipsometry. The thicknesses and refractive indices of the films (spin-cast 152 and adsorbed layers) were determined using a variable-angle spectroscopic ellipsometer (J.A. 153 Woollam Co., Inc., Lincoln, NE, USA) in air. The angle-of-incidence ranged between 64 and $15475^{\circ}$ depending on the substrate and the particular system, and the wavelength ranged across the 155 visible region from 400 to $800 \mathrm{~nm}$. The central area of the film, where it was most smooth and 156 uniform, was probed. Spectra were analysed using commercial software (WVASE 32, J.A. 157 Woollam Co., Inc.). All films were stored at in a desiccator prior to their analysis in order to 158 ensure reproducibility of the thickness measurements. (The copolymer thickness was found to 159 increase with the relative humidity, but the initial thickness was recovered when placed in a 160 low humidity atmosphere.)

161 Infrared ellipsometry spectra were obtained in air using a Fourier transform IR ellipsometer 162 (Model GESP5-FTIR, SOPRA Sa., Bois-Colombes, France) at an angle-of-incidence of $65^{\circ}$. 163 The spectra were obtained with a spectral resolution of $4 \mathrm{~cm}^{-1}$ over a range from 4000 to 650 $164 \mathrm{~cm}^{-1}$. A typical measurement used eight scans for each spectrum, and 32 spectra were averaged 165 to yield a higher signal-to-noise ratio.

166 The $\Psi$ and $\Delta$ parameters obtained from the IR ellipsometry spectra were then converted to 167 real and imaginary optical density values using these relations:

$$
\operatorname{Re} D=\ln \left(\tan \Psi_{o} / \tan \Psi_{f}\right)
$$

and

$$
\operatorname{Im} D=i\left(\Delta_{o}-\Delta_{f}\right)
$$


where the subscript $o$ and $f$ refer to the original surface and to the final surface after the deposition of a surface layer. Only the $\operatorname{Re} D$ data will be presented here because, as shown earlier, ${ }^{27}$ for isotropic organic films on metallic substrates over a limited range of thicknesses, the $\tan \Psi$ IR ellipsometry spectra are similar in shape to ordinary absorption spectra.

Infrared Spectroscopy. IR spectra were also obtained from the mucin in bulk form rather than in a thin film. $\mathrm{KBr}$ and mucin were separately crushed to create fine powders. $\mathrm{KBr}$ powder was dried in a fan oven at $40{ }^{\circ} \mathrm{C}$, and the mucin was dried at room temperature in a desiccator containing silica gel for $24 \mathrm{~h}$. The two powders were mixed, and then sufficient pressure was applied to make a solid pellet. FTIR spectrometer (Perkin Elmer system 2000 FTIR, Waltham, Ma., USA) was used to obtain the absorption spectra at a resolution of $2 \mathrm{~cm}^{-1}$ and averaging 128 scans.

Regularized Method of Deconvolution. The theory of the regularized method of deconvolution has been described in detail elsewhere. ${ }^{33,34}$ The deconvolution method was applied to the measured $\operatorname{Re} D$ ellipsometry data. The deconvolution parameters - the Lorentzian contour half-width $\left(W_{L}\right)$ and the passband value of the deconvolution filter $(L)$ for the ellipsometry spectra - were set for the various spectral regions as follows: $W_{L}=16.0-17.0$ $\mathrm{cm}^{-1}\left(1800-1700,1600-1000 \mathrm{~cm}^{-1}\right), W_{L}=20-22 \mathrm{~cm}^{-1}\left(1700-1600 \mathrm{~cm}^{-1}\right)$, and $L=0.12 \mathrm{~cm}$. For the IR absorption spectrum in the $1700-1600 \mathrm{~cm}^{-1}$ range, $W_{L}$ was set $26 \mathrm{~cm}^{-1}$ and $L$ was 0.1 $\mathrm{cm}$. These values of the deconvolution parameters were chosen to provide the highest resolution enhancement in the experimental spectra at the given signal-to-noise ratio. The reliability of the results of deconvolution was confirmed by coincidence of the deconvolved spectra of several samples of the same compounds.

\section{RESULTS AND DISCUSSION}

Visible Ellipsometry of Mucin Layers Adsorbed on Polymer Surfaces. Spectroscopic ellipsometry in visible range was used to examine the thicknesses of adsorbed mucin layers on both the PAA-b-PMMA copolymer and PMMA surfaces on Si substrates. While PMMA is relatively hydrophobic, the PAA- $b$-PMMA copolymer is amphiphilic ${ }^{35,36}$ owing to the presence of a hydrophilic poly(acrylic acid) block.

Figure 1 shows, as an example, the ellipsometry spectra obtained from mucin layers adsorbed at a $\mathrm{pH}$ of 3 and 10 onto a PAA- $b$-PMMA film in comparison to the original copolymer film. There are obvious differences in the spectra that are attributed to the 
202 adsorption of mucin, and an effect of $\mathrm{pH}$ is also readily apparent in the spectra. (In all cases,

203 the samples had been dried in a desiccator prior to the measurement to ensure a fair 204 comparison.)

205 Analysis of the ellipsometry data has been performed with the use of an effective medium 206 approximation (EMA). ${ }^{37}$ This model allows the calculation of the refractive index, $n$, of a 207 composite of one phase dispersed in another. In our case, the mucin coatings were described as 208 a composite of dense mucin and air voids. The refractive index therefore provides an indication 209 of the void content (or density). The refractive index of dense (void-free) mucin was obtained 210 from an independent measurement on a thick, dry layer (ca. $200 \mathrm{~nm}$ ). The dependence of $n$ on 211 the wavelength, $\lambda$, was described using the Cauchy equation, $n=A+B / \lambda^{2}$, where $A$ was found 212 experimentally to be 1.61 and $B$ found to be $0.001 \mu \mathrm{m}^{2}$.

213 The ellipsometry spectra were modeled using a standard slab model ${ }^{24,28,30}$ under the 214 assumption of the isotropy of the optical properties of the polymer and mucin films. The 215 thicknesses and refractive indices of the silicon's native oxide layer and the polymer films were 216 obtained in preliminary measurements. After the mucin adsorption, the model consisted of a 217 mucin layer of unknown thickness, the polymer film, the native oxide layer, and silicon. Note 218 that the application of a similar multilayer optical model for the evaluation of ellipsometry 219 spectra of protein layers adsorbed on polymer surfaces, as shown earlier, ${ }^{38,39}$ provides 220 sufficiently reliable characterization and good resolution.

221 The quantitative results of data modeling (Table I) reveals differences in thickness in the very thin layers of biomolecules. Table I indicates that the thickness of adsorbed mucin layers on the more hydrophobic PMMA surface is in the range expected for a molecular monolayer coating (about $4 \mathrm{~nm}$ ), and which is consistent with the data reported by others. ${ }^{6}$ The thickness of BSM layers on PMMA does not vary with $\mathrm{pH}$. (The reported thickness values are an 226 average for the layer.)

227 At all $\mathrm{pH}$ values, however, the thickness of BSM layers adsorbed on PAA-b-PMMA is 228 greater than found on PMMA. Furthermore, unlike the case for PMMA, the average layer 229 thickness on the PAA-b-PMMA is strongly dependent on $\mathrm{pH}$. At a $\mathrm{pH}$ of 3 , the mucin 230 thickness is more than double that at higher $\mathrm{pH}$ values. There is not any significant difference 231 in the BSM layers thickness when adsorbed at $\mathrm{pH}$ values of 7 and 10.

232 The values obtained for the void fractions rely on the assumption that a thick mucin layer is 233 fully dense, but in any case, the data in Table I clearly indicate trends in the layer densities. 234 The analysis of the ellipsometry data shows that the percentage of voids in the BSM coating on 235 PAA- $b$-PMMA is the smallest at acidic $\mathrm{pH}$, and slightly increases under neutral conditions 
236 (Table I). From this analysis, it can be concluded that the most complete mucin coverage on the 237 PAA- $b$-PMMA surface is obtained at a $\mathrm{pH}$ of 3.

238 On the basis of previous studies of mucin adhesion on PAA, ${ }^{3,7,8}$ we suspect that the 239 observed increase in the thickness of adsorbed BSM layers on the copolymer surface at a pH of

2403 can be explained by an increase in the proportion of protonated carboxyl $(\mathrm{COOH})$ groups of 241 mucin and PAA able to form hydrogen bonds. However, details of the particular hydrogen 242 bonding mechanism, and whether the mucin's amide groups are contributing, cannot be 243 obtained from these results alone, which prompted IR ellipsometry analysis.

244 Proteins are known to undergo conformational changes and re-orientation upon adsorption 245 on various surfaces. ${ }^{38-43}$ These changes are influenced by the hydrophobicity of the surface, 246 charge, protein concentration, $\mathrm{pH}$ and various other chemical and physical factors. In 247 particular, it was revealed ${ }^{42,43}$ that the protein aggregation at solid interfaces correlates with an 248 increase in the content of $\beta$-sheets in the secondary structure. The extent of conformational 249 changes of some proteins was found ${ }^{41-43}$ to be dependent on the amount of adsorption. 250 However, unlike the situation for proteins, there are scarce reports in the literature ${ }^{17,18}$ about the 251 secondary structure of the protein backbone of BSM adsorbed on polymer surfaces. However, 252 IR ellipsometry is shown hereafter to determine the conformational changes caused by the 253 adsorption of BSM on PAA- $b$-PMMA surface.

254 In investigating the adhesion and conformational changes of mucin on an amphiphilic 255 diblock copolymer surface we analysed $\tan \Psi$ IR ellipsometry spectra of BSM/PAA- $b$ 256 PMMA double layers. The ellipsometry spectra of mucin layers and copolymer films on gold 257 were used as a reference to reveal characteristic changes in the spectra of bilayers due to 258 adhesion.

259 IR Ellipsometry Spectra of Mucin Layers and Copolymer Films. The IR ellipsometry 260 spectra of mucin layers on gold (Fig. 2a) are consistent with what is known about the structure 261 of mucin. The spectra are characterized by the presence of Amide I $\left(1657 \mathrm{~cm}^{-1}\right)$, Amide II $262\left(1540 \mathrm{~cm}^{1}\right)$, and Amide III $\left(1240 \mathrm{~cm}^{-1}\right)$ bands and by several components in the 1200-1000 $263 \mathrm{~cm}^{-1}$ range, which mainly originate from the carbohydrate side chains of mucin. ${ }^{10,44-46}$

264 The $\mathrm{pH}$ of the mucin solution is found to affect the spectra of the layers that are deposited 265 from them. Some characteristic differences are observed between the spectra of mucin layers 266 deposited at a pH of 3 and 7 (Fig. 2a). Specifically, increasing the $\mathrm{pH}$ value from 3 to 7 leads to 267 a decrease in the intensity of the carbonyl band $(\mathrm{v}(\mathrm{C}=\mathrm{O}))$ near $1720 \mathrm{~cm}^{-1}$, which is attributed to 268 the $\mathrm{COOH}$ groups of the mucin's sialic acid. ${ }^{46}$ Together with this, an increase in the absorption 269 near 1556 and $1400 \mathrm{~cm}^{-1}$ corresponding, respectively, to the antisymmetric $\mathrm{v}_{\mathrm{a}}\left(\mathrm{COO}^{-}\right)$and 
symmetric $v_{\mathrm{s}}\left(\mathrm{COO}^{-}\right)$stretching vibrations of carboxylate groups is observed with increasing $\mathrm{pH}$.

272 The original ellipsometry spectrum of the PAA-b-PMMA copolymer film shows the 273 presence of a characteristic carbonyl band at $1732 \mathrm{~cm}^{-1}, \mathrm{CH}_{2}$ deformation at $1450 \mathrm{~cm}^{-1}$, and the

274 broad absorption bands in the $1300-1150 \mathrm{~cm}^{-1}$ range, mainly related to the $\mathrm{CO}$ and $\mathrm{CC}$ 275 stretching vibrations of the copolymer's side groups (Fig. 2b). ${ }^{47-49}$

\section{SPECTROSCOPIC EVIDENCE FOR HYDROGEN BOND FORMATION IN THE} BSM/PAA- $b$-PMMA BILAYER

Carbonyl Stretching Region. Bilayers of mucin deposited onto copolymer films on gold were used to gain insight into the bonding mechanism between the two substances and conformational changes induced by their interactions. It is particularly useful to compare the spectra from the bilayer to the "sum spectra" obtained through the mathematical combination of the relevant spectra of the individual layers. Because of the relatively weak contribution of the $v(C=O)$ band of carboxyl groups of mucin near $1720 \mathrm{~cm}^{-1}$ in comparison to the band of the copolymer, the sum spectra in the carbonyl stretching range are virtually identical to each other at the two $\mathrm{pH}$ values (Figs. $2 \mathrm{c}$ and $3 \mathrm{a}$ ). In the original experimental spectra of mucin/copolymer bilayers, a low-frequency shift and a change in the shape of the $v(C=O)$ band of the copolymer is observed when comparing to the sum spectra (Fig. 3a).

Further important information is provided by the deconvolution of the spectra (Fig. 3b). It is revealed that the asymmetric carbonyl band of the copolymer consists of two components with different intensities. The component at $1736 \mathrm{~cm}^{-1}$ is attributed to the $\mathrm{v}(\mathrm{C}=\mathrm{O})$ stretching vibrations of "free" (i.e. non-hydrogen-bonded) ester and carboxyl groups in the PMMA and 293 PAA units of the copolymer, respectively. On the other hand, the component at $1712 \mathrm{~cm}^{-1}$ is 294 known to be a characteristic of the hydrogen-bonded $\mathrm{COOH}$ groups in PAA. ${ }^{47,48}$ There are marked differences in the deconvolved spectra of the bilayers in comparison to the sum of the spectra of the single films. In particular, there is a shift towards lower frequencies and a 297 broadening of the band corresponding to the hydrogen-bonded $\mathrm{C}=\mathrm{O}$ groups of the copolymer at $2981712 \mathrm{~cm}^{-1}$ (Fig. 3b). This fact is indicative of the formation of hydrogen bonds between the 299 copolymer and mucin macromolecules. ${ }^{48}$ It is worth noting that the band of the "free" $\mathrm{C}=\mathrm{O}$ 300 groups is also shifted in the deconvolved spectra of bilayers. This result could reflect changes 301 in the chemical environment around the carbonyl groups of the copolymer because of 302 entanglements with mucin chains.

303 Mucins in aqueous solution are known to have a "bottle-brush" structure with hydrophilic 
304 carbohydrate branch chains exposed at the outermost surface. ${ }^{3}$ Therefore carboxyl groups in

305 the mucin side chains are considered to be the most probable sites for hydrogen bonding upon

306 mucoadhesion. Furthermore, it has been shown elsewhere that the surface rearrangement of

307 amphiphilic block copolymers also occurs when in contact with water. ${ }^{35,36}$ Hydrophilic blocks

308 tend to locate at the surface, whereas hydrophobic segments are buried under the surface. It

309 follows that upon exposure of the mucin solution, the surface of PAA-b-PMMA copolymer

310 film will be enriched with $\mathrm{COOH}$ groups of the hydrophilic PAA block. Hence, the formation

311 of hydrogen bonds is favored in the double-layer system via the $\mathrm{COOH}$ groups of both the

312 mucin and the copolymer. Figure 4 shows the proposed types of hydrogen bonding.

313 Some differences between the deconvolved spectra of the bilayers at $\mathrm{pH}$ values of 3 and 7

314 can be seen in Fig. 3b, namely, the contribution of hydrogen-bonded $\mathrm{C}=\mathrm{O}$ groups to the

315 complex $\mathrm{v}(\mathrm{C}=\mathrm{O})$ band contour is lower at a $\mathrm{pH}$ of 7 in comparison to a $\mathrm{pH}$ of 3 . This result

316 suggests the participation of terminal $\mathrm{COOH}$ groups of sialic acid in hydrogen-bonding

317 interactions during mucoadhesion. It worth noting that the complex structure of the $v(C=O)$

318 band in the spectra of bilayers prevents reliable quantitative estimate of the extent of hydrogen

319 bonding of mucin and copolymer macromolecules at different $\mathrm{pH}$ values.

320 It is well-known ${ }^{3,8}$ that at a $\mathrm{pH}$ of 7 most of the carboxyl groups of sialic acid (with a $\mathrm{pKa}$ 321 of 2.6) are ionized and consequently are not available for hydrogen bonding. Likewise, the

322 surface properties of amphiphilic copolymer films have been shown to be greatly influenced by

$323 \mathrm{pH}^{35,36}$ At $\mathrm{pH}$ values higher than the $\mathrm{pKa}$ of 4 to 5,9 deprotonation of the $\mathrm{COOH}$ groups of

324 PAA blocks at the interface occurs, which leads to a electrostatic repulsion between negatively-

325 charged PAA and mucin groups. Due to the repulsion of uncoiled polymer chains, the density

326 of chain packing decreases, which can result in reducing interdiffusion and molecular

327 entanglements. ${ }^{8}$ In accord with this concept, a more pronounced low-frequency shift at a $\mathrm{pH}$ of

3287 for the band of "free" $\mathrm{C}=\mathrm{O}$ groups at $1736 \mathrm{~cm}^{-1}$ (Fig. 3b) can be attributed to the greater

329 changes in the structure of the copolymer chain packing because of the strong repulsion of

330 negatively-charged, uncoiled PAA blocks.

331 Thus, the IR ellipsometry results confirm that the considerable increase in the thickness of 332 the adsorbed mucin layers at $\mathrm{pH} 3$, as compared to $\mathrm{pH} 7$, revealed by visible ellipsometry, can 333 be explained by a larger extent of hydrogen bonding between mucin and copolymer 334 macromolecules. Interestingly, a similar tendency for the adsorption of a protein (bovine serum 335 albumin) on a PAA brush layer to decrease at $\mathrm{pH}$ values higher than 6 has been revealed 336 elsewhere. $^{50}$

337 It is worth noting that, according to visible ellipsomery data, the average thickness of 
mucin layer adsorbed on the copolymer surface at $\mathrm{pH} 7$ and 10, when the carboxyl groups are ionized, is two times larger than that on PMMA. This observation can be attributed to the fact that introducing PAA blocks increases interdiffusion at the mucin/copolymer interface and the extent of molecular chain entanglements, ${ }^{8,9}$ which results in more mucin adsorption on PAA- $b$ PMMA copolymer surfaces, as compared to PMMA.

1350-1000 $\mathrm{cm}^{-1}$ Region. In the original IR ellipsometry spectrum of copolymer in the $1300-1150 \mathrm{~cm}^{-1}$ range, as already mentioned, a broad contour with maxima at about 1256 and $1192 \mathrm{~cm}^{-1}$ is observed (Fig. 2b). The vibrational bands in this region can be mainly assigned to the combined $\mathrm{CO}$ and $\mathrm{CC}$ stretching vibrations in the copolymer's ester and carboxyl side

347 groups. ${ }^{47-49}$ These bands are known to be very sensitive both to conformational changes in polymer macromolecules and to hydrogen bonding. ${ }^{47,48}$

349 With the application of the deconvolution method, the fine structure of the complex copolymer bands in 1300-1150 $\mathrm{cm}^{-1}$ range has been revealed (Fig. 5b). The existence of four additional new components has been established (1292, 1271, 1167, and $\left.1157 \mathrm{~cm}^{-1}\right)$.

352 Multiplicity of the bands in this range can be related to different conformers of side groups in copolymer macromolecules, as has been reported earlier for PMMA. ${ }^{49}$ According to the literature for PMMA, ${ }^{49}$ in the $1300-1220 \mathrm{~cm}^{-1}$ range, the absorption bands mainly arise from antisymmetric $\mathrm{v}_{\mathrm{a}}(\mathrm{C}-\mathrm{C}-\mathrm{O})$ stretching vibrations of ester groups in the trans- and cisconformations, whereas in the $1220-1150 \mathrm{~cm}^{-1}$ range, the bands are associated with $\mathrm{v}_{\mathrm{a}}(\mathrm{C}-\mathrm{O}-\mathrm{C})$ vibrational modes of different rotational isomers of ester groups of the PMMA block. Moreover, $\mathrm{CO}$ stretching vibrations coupled with $\mathrm{OH}$ in-plane bending of carboxyl groups of PAA segments contribute greatly to the absorption in the given intervals. ${ }^{47,48}$

In Fig. 5 it is seen that most of the bands of the copolymer in the $1350-1150 \mathrm{~cm}^{-1}$ range appear in the sum spectra. Comparing the experimental and the sum spectra, pronounced differences are observed in the $1300-1150 \mathrm{~cm}^{-1}$ range in the deconvolved spectra (Fig. 5c) that were less apparent in the original spectra (Fig. 2c). Thus, a redistribution of the relative

365 the $1292 \mathrm{~cm}^{-1}$ component are all observed in the experimental spectra but not in the sum 366 spectra. In accordance with the assignments of the bands in this range, the observed spectral

367 differences could be indicative of changes in hydrogen bonding of carboxyl groups in PAA segments caused by mucoadhesion. Furthermore, some changes in the conformational states of side groups of the copolymer induced by entanglements and interactions with the mucin 370 macromolecules could have occurred. That is, an increase in the relative intensity of the band 371 near $1271 \mathrm{~cm}^{-1}$ can be related to a larger content of trans conformations of ester groups in the 
372 PMMA block. A similar tendency for conformational changes of the side-chain ester groups

373 has been previously reported for mixing in thin polymer double layers. ${ }^{27}$

374 In the $1150-1000 \mathrm{~cm}^{-1}$ region, the bands originating from the carbohydrate residues of 375 mucin contribute significantly to the sum spectra (Fig. 5a,c). The spectral alterations in the

376 given range are consistent with those observed in the above regions and indicate the formation

377 of hydrogen bonds between carboxyl groups of mucin and copolymer macromolecules.

Effects of $\mathrm{pH}$ are also observed in the spectral changes in the $1350-1000 \mathrm{~cm}^{-1}$ range. Some differences between the spectra of bilayers at the two $\mathrm{pH}$ values are found, because of vibrations involving $\mathrm{COOH}$ groups of PAA and mucin. This observation confirms the conclusions drawn above regarding the formation of hydrogen bonding in the bilayer via $\mathrm{COOH}$ groups of copolymer and mucin (Fig. 4).

As mentioned in the Introduction, according to IR spectroscopy data from mucin/PAA mixtures, ${ }^{10}$ hydrogen bonds can be formed between carboxyl groups of PAA and amide groups of mucin. However, our analysis here does not support this mechanism. If the mucin's amide groups were solely contributing to the hydrogen bonding, then changes in the bands related to the vibrations of $\mathrm{COOH}$ groups of mucin would not have been observed.

\section{SECONDARY STRUCTURE OF MUCIN LAYERS ON GOLD AND COPOLYMER}

\section{SURFACES}

Amide I Region. The amide I band is mainly the result of stretching vibrations of hydrogen-bonded $\mathrm{C}=\mathrm{O}$ groups in the protein backbone of mucin with some contribution from the stretching vibrations of $\mathrm{CN}$ groups. ${ }^{10,18}$ The band is well-known to be very sensitive to the conformational changes in the secondary structure of protein molecules and to the degree of hydrogen bonding. ${ }^{18,51-53}$ Study of the amide I band therefore provides important insights to any conformational changes and bonding that might occur during mucoadhesion.

The protein backbone of mucin is arranged into several distinct regions. ${ }^{1}$ Glycosylated peptide blocks are rich in serine, threonine and proline, whereas unbranched peptide blocks or regions with little glycosylation have a high content of cysteine. The domains that are rich in cysteine are believed to be globular. ${ }^{1,2}$

404 absorption IR spectrum of the mucin powder (Fig. 6a, curve 1) the amide I band is observed

The mucin macromolecules in solution are shown to have a mainly random-coil structure at $\mathrm{pH} \geq 4$ and anisotropic extended conformation at $\mathrm{pH}<4 .{ }^{11}$ It has been found elsewhere that the secondary structure of BSM in the solid state differs from that in solution. ${ }^{18}$ In the original near $1650 \mathrm{~cm}^{-1}$. With the use of the deconvolution method, a number of individual components 
406 of the given band have been revealed (Fig. 6a, curve 2). According to the literature, ${ }^{43,51-53}$ the 407 observed components could reflect the presence of $\beta$-turns $\left(1661 \mathrm{~cm}^{-1}\right), \alpha$-helix $\left(1649 \mathrm{~cm}^{-1}\right)$, 408 random coil $\left(1641 \mathrm{~cm}^{-1}\right)$ and $\beta$-sheets $\left(1694\right.$ and $\left.1632 \mathrm{~cm}^{-1}\right)$ in the secondary structure of the 409 protein backbone. The given secondary structure elements are consistent with the complex 410 amino acid composition of polypeptide chains of mucin. ${ }^{1-3}$

411 Comparison of the results of deconvolution of the amide I band in the dry bulk powder and 412 in the layers (Fig. 6) shows that upon mucin adsorption, significant conformational changes of 413 the protein backbone occur. The amide I band observed in the original ellipsometry spectra of 414 the mucin layers on gold (Fig. 6b, curve 1) is shifted to $1657 \mathrm{~cm}^{-1}$, as compared to the 415 absorption IR spectrum of the mucin powder, which indicate some changes in the secondary 416 structure of the protein backbone of mucin upon adsorption on the gold surface. For the BSM 417 on gold, the results of deconvolution suggest predominantly an $\alpha$-helix secondary structure of 418 the protein backbone $\left(1657 \mathrm{~cm}^{-1}\right)$ with some noticeable contribution of $\beta$-sheets $\left(1632 \mathrm{~cm}^{-1}\right)$ 419 and $\beta$-turns (broadly around $1670 \mathrm{~cm}^{-1}$ ). ${ }^{43,51-53}$ Some differences observed in the 1675-1660 $420 \mathrm{~cm}^{-1}$ range for the amide I band of the mucin layers on gold could reflect a larger contribution 421 of $\beta$-turns in the secondary structure at a $\mathrm{pH}$ of 7 compared to the $\mathrm{pH}$ of 3 (Fig. 6b, curve 1).

422 The fine structure of the amide I band of mucin layers on gold, revealed as a result of 423 deconvolution, is compared to that of mucin on the copolymer surface in Fig. $6 \mathrm{~b}$. In the 424 deconvolved spectra of the mucin on the copolymer surface at the different $\mathrm{pH}$ values (Fig. 6b, 425 curve 2), alterations in the relative intensities of the individual components of complex amide I 426 band, as compared to the mucin layers on gold, are revealed. The band associated with $\beta$-sheets 427 (around $1630 \mathrm{~cm}^{-1}$ ) is stronger for the bilayers compared to the mucin layers on the more 428 hydrophobic gold surface. Furthermore, the maximum of the main component corresponding to $429 \alpha$-helix secondary structure is shifted towards lower frequencies in the case of the bilayers. 430 This observation indicates that the adhesion of BSM on the PAA- $b$-PMMA is accompanied by 431 changes in the secondary structure of the protein backbone. Results of deconvolution indicate 432 an increase of the proportion of $\beta$-sheets together with a decrease in the amount of $\beta$-turns. 433 This suggests a more unfolded rigid structure of the protein backbone of mucin 434 macromolecules after their adhesion on the copolymer surface. It has been shown previously ${ }^{40-}$ $435{ }^{42}$ that the increase in the amount of $\beta$-structures can be indicative of the aggregation of 436 proteins at the interface and of the formation of a stronger network. In Fig. $6 \mathrm{~b}$ it is seen that the 437 proportion of $\beta$-sheets is slightly higher at a $\mathrm{pH}$ of 3 as compared to a $\mathrm{pH}$ of 7 . It follows that 438 the larger amount of the BSM adsorbed on the copolymer surface at a $\mathrm{pH} 3$, revealed by visible 
ellipsometry, correlates with the higher content of $\beta$-sheets.

440 It is worth noting that an increase in the content of $\beta$-sheets in secondary structure has also 441 been reported earlier for BSM irreversibly adsorbed on hydrogel surfaces. ${ }^{18}$ In other works on 442 protein adsorption ${ }^{41-43}$ it was found that a greater amount of adsorbed protein macromolecules 443 leads to greater changes in their secondary structure. Our results reveal a similar correlation 444 between the conformational changes of the protein backbone of mucin molecules and the 445 amount of mucin adsorbed onto the copolymer surface.

446 Amide II Region. The amide II band (Fig. 2a,7a) is mainly related to an in-plane NH 447 bending coupled with C-N and C-C stretching vibrations. ${ }^{18,51-53}$ It has been shown ${ }^{18}$ that upon 448 changes in conformation and hydrogen bonding of the protein backbone of mucin, one can 449 observe a change in the halfwidth of this band and a shift in the direction opposite to that 450 observed for the amide I band.

451 In Fig. 7a it is seen that Amide II band of the mucin layers $\left(1540 \mathrm{~cm}^{-1}\right)$ is overlapped with 452 the $\mathrm{pH}$-sensitive component near $1556 \mathrm{~cm}^{-1}$ related to the antisymmetric $\mathrm{v}_{\mathrm{a}}\left(\mathrm{COO}^{-}\right)$stretching 453 vibrations of the carboxylate groups.

454 Fig. 7c reveals significant differences between the deconvolved ellipsometry spectra of 455 bilayers at the two different $\mathrm{pH}$ values. The sum spectra, on the other hand, do not show any 456 difference with $\mathrm{pH}$, indicating that changes are brought on by the bilayer formation. In the 457 ellipsometry spectrum of the bilayer at a $\mathrm{pH}$ of 7 , a considerable broadening of the spectral 458 bands near 1556 and $1400 \mathrm{~cm}^{-1}$ occur, which might indicate changes in the electronic 459 environment of the carboxylate groups because of the repulsion of like-charged chains of 460 mucin and PAA. However, Fig. 7c shows that a significant broadening of the spectral contour 461 in the Amide II region (1580-1525 $\left.\mathrm{cm}^{-1}\right)$ is also observed at a $\mathrm{pH}$ of 3, when most of the $462 \mathrm{COOH}$ groups are protonated. This allows us to draw the conclusion that the spectral 463 alterations revealed in the Amide II region probably reflect changes in the conformations and 464 hydrogen bonding of the protein backbone of mucin upon adhesion. 
Using spectroscopic ellipsometry we have determined the thickness of mucin layers adsorbed onto PAA-b-PMMA copolymer and PMMA surfaces under acidic and neutral $\mathrm{pH}$ conditions. The average thickness of the mucin layers on the copolymer surface - but not on PMMA - was determined to be $\mathrm{pH}$ dependent. The mucin thickness is more than two times greater when deposited at a $\mathrm{pH}$ of 3 compared to neutral conditions. The most complete mucin coating was obtained on the PAA- $b$-PMMA copolymer surface at this $\mathrm{pH}$, and this finding can be exploited when preparing coatings to reduce bacterial adhesion.

With the use of IR ellipsometry and the regularized method of deconvolution, spectroscopic evidence for the formation of hydrogen bonds between the copolymer and mucin macromolecules in the BSM/copolymer double-layer system on gold has been found. The IR ellipsometry data, in agreement with the visible ellipsometry analysis, reveal the $\mathrm{pH}$ dependence of adhesion of mucin to the copolymer surface. There is an increase in the amount of hydrogen-bonded carboxyl groups in mucin deposited at a $\mathrm{pH}$ of 3 . There is no evidence that the amide groups of the mucin participate in hydrogen bonding with the $\mathrm{COOH}$ groups of PAA, although this mechanism has been proposed in the literature.

Using the regularized method of deconvolution, it has been found that the process of adhesion of mucin on the PAA- $b$-PMMA surface, in comparison to the more hydrophobic gold surface, is accompanied by changes in the secondary structure of the protein backbone. The proportion of $\beta$-sheets therewith increases, which indicates a more unfolded, aggregated structure of the mucin macromolecules after adhesion. The conformational changes in the protein backbone of mucin are found to be $\mathrm{pH}$ dependent. A slightly higher proportion of $\beta$ sheets in the secondary structure of the protein backbone at a $\mathrm{pH}$ of 3 has been revealed, which correlates with the greater amount of mucin adsorbed on the copolymer surface at this $\mathrm{pH}$. The structure of biomacromolecules and adhesive interactions in double layers. We have shown that spectroscopic ellipsometry is an effective tool for studies of bioadhesion in multi-layered systems. The use of the deconvolution method enables a more reliable identification of the deconvolution results of IR ellipsometry spectra allow us to conclude that some changes in the conformational states of the side groups of the copolymer, caused by entanglements and interactions with the mucin macromolecules, occur upon adhesion.

This work is the first detailed use of IR ellipsometry to determine the conformational structural changes and molecular interactions due to the adhesion of biomolecules to polymer surfaces. 


\section{ACKNOWLEDGMENTS}

501 We are grateful to the Ministry of Higher Education of Saudi Arabia for a Ph.D scholarship 502 for I.A.B., who performed all the experiments reported here, and to the Royal Society for a 503 travel grant to N.A.N. to visit the University of Surrey. We acknowledge assistance from Dr. 504 G. Cavalli (University of Surrey) in the IR spectroscopy experiments.

505

506 
1. R. Bansil and B. S. Turner, Current Opinion in Colloid \& Interface Sci. 11, 164 (2006).

2. S. E. Harding, S. S. Davis, M. P. Deacon, and I. Fiebrig, Biotechnol. Genetic Eng. Rev. 16, 41 (1999).

3. N. A. Peppas and Y. Huang, Adv. Drug Delivery Rev. 56, 1675 (2004).

4. J. D. Smart, Adv. Drug Delivery Rev. 57, 1556 (2005).

5. L. Shi, R. Ardehali, K. D. Caldwell, and P. Valint, Colloids Surf., B 17, 229 (2000).

6. L. Shi, R. Ardehali, P. Valint, and K. D. Caldwell, Biotechn. Lett. 23, 437 (2001).

7. H. Park and J. R. Robinson, Pharm. Res. 4, 457 (1987).

8. J. Cleary, L. Bromberg, E. Magner, Langmuir 20, 9755 (2004).

9. E. Jabbari, N. Wisniewski, and N. A. Peppas, J. Controlled Release 26, 99 (1993).

10. M. M. Patel, J. D. Smart, T. G. Nevell, R. J. Ewen, P. J Eaton, and J. Tsibouklis, Biomacromolecules 4, 1184 (2003).

11. X. Cao, R. Bansil, K. R. Bhaskar, B. S. Turner, J. T. LaMont, N. Niu, and N. H. Afdhal, Biophys. J. 76, 1250 (1999).

12. Z. Hong, B. Chasan, R. Bansil, B. S. Turner, K. R. Bhaskar, and N. H. Afdhal, Biomacromolecules 6, 3458 (2005).

13. M. P. Deacon, S. McGurk, C. J. Roberts, P. M. Williams, S. J. B. Tendler, M. C. Davies, S. S. Davis, and S. E. Harding, Biochem. J. 348, 557 (2000).

14. N. V. Efremova, Y. Huang, N. A. Peppas, and D. E. Leckband, Langmuir 18, 836 (2002).

15. J. McColl and G. E. Yakubov, J. J. Ramsden, Langmuir 24, 902 (2008).

16. J. McColl and G. E. Yakubov, J. J. Ramsden, Langmuir 23, 7096 (2007).

17. S. Lee, M. Müller, K. Rezwan, and D. Spencer, Langmuir 21, 8344 (2005).

18. E. J. Castillo, J. L. Koenig, J. M. Anderson, and N. Jentoft, Biomaterials 7, 9 (1986).

19. K. Hinrichs, M. Gensch, and N. Esser, Appl. Spectrosc. 59, 272A (2005).

20. T. Schram and H. J. Terryn, Electochem. Soc. 148, F12 (2001).

21. K. Hinrichs, A. Röseler, and K. Roodenko, J. Rappich Appl. Spectrosc. 62, 121 (2008).

22. H. G. Tompkins, T. Tiwald, C. Bungay, and A. E. Hooper, J. Phys. Chem. B 108, 3777 (2004).

23. C. L. Bungay, T. E. Tiwald, D. W. Thompson, M. J. DeVries, J. A. Woollam, and J. F. Elman, Thin Solid Films 313, 713 (1998).

24. T. R. E. Simpson, Z. Tabatabaian, C. Jeynes, B. Parbhoo, and J. L. Keddie, J. Polym. Sci. Pt. A. Polym. Chem. 42, 1421 (2004). 
25. T. R. E. Simpson and J. L. Keddie, J. Adhesion 79, 1207 (2003).

26. C. Bungay and T. E. Tiwald, Thin Solid Films 455, 272 (2004).

27. N. A. Nikonenko, K. Hinrichs, E. H. Korte, J. Pionteck, and K.-J. Erichhorn, Macromolecules 37, 8661 (2004).

28. P. Duckworth, H. Richardson, C. Carelli, and J. L. Keddie, Surf. Interf. Anal. 36, 33 (2005).

29. Y. Mikhaylova, L. Ionov, J. Rappich, M. Gensch, N. Esser, S. Minko, K.-J. Eichhorn, M. Stamm, and K. Hinrichs Anal. Chem. 79, 7676 (2007).

30. B. J. Lincoln, T. R. E. Simpson, and J. L. Keddie, Coll. Surf. B Bionterfaces 33, 251 (2004).

31. E. Garcia-Caurel, B. Drevillon, A. De Martino, and L. Schwartz, Appl. Optics 41, 7339 (2002).

32. K. Hinrichs, M. Gensch, N. Esser, U. Schade, J. Rappich, S. Kröning, M. Portwich, and R. Volkmer, Anal. Bioanal. Chem. 387, 1823 (2007).

33. D. K. Buslov and N. A. Nikonenko, Appl. Spectrosc. 51, 666 (1997).

34. D. K. Buslov and N. A. Nikonenko, Appl. Spectrosc. 52, 613 (1998).

35. I. Luzinov, S. Minko, and V. V. Tsukruk, Prog. Polym. Sci. 29, 635 (2004).

36. M. Callewaert, J.-F. Gohy, C. C. Dupont-Gillain, L. Boulangé-Petermann, and P. G. Rouxhet, Surf. Sci. 575, 125 (2005).

37. D. E Aspnes, J. B. Theeten, and F. Hottier, Phys. Rev. B 20, 3292 (1979).

38. C. Werner, K.-J. Eichorn, K. Grundke, F. Simon, W. Grählert, H.-J. Jacobasch Colloids and Surfaces A: Physicochem. Eng. Aspects 156, 3 (1999).

39. Y. Mikhaylova, V. Dutschk, M. Müller, K. Grundke, K.-J. Eichorn, B. Voit, Colloids and Surfaces A: Physicochem. Eng. Aspects 297, 19 (2007).

40. R. J. Green, I. Hopkinson, and R. A. L. Jones, Langmuir 15, 5102 (1999).

41. A. H. Martin, M. B. J. Meinders, M. A. Bos, M. A. Cohen Stuart, and T. van Vliet, Langmuir 19, 2922 (2003).

42. A. Sethuraman, G. Vedantham, T. Imoto, T. Przybycien, and G. Belfort, Proteins: Str., Func., Bioinform. 56, 669 (2004).

43. R. Ishiguro, Y. Yokoyama, H. Maeda, A. Shimamura, K. Kameyama, and K. Hiramatsu, J. Colloid Interface Sci. 290, 91 (2005).

44. D. R. Skingsley, A. J. White, and A. J. Weston, Moll. Stud. 66, 363 (2000).

45. C. D. Bavington, R. Lever, B. Mulloy, M. M. Grundy, C. P. Page, N. V. Richardson, and J. D. McKenzie, Comparative Biochem. Physiol., B 139, 607 (2004). 
46. M. Khajehpour, J. L. Dashnau, and J. M. Vanderkooi, Anal. Biochemistry 348, 40 (2006).

47. J. Dong, Y. Ozaki, and K. Nakashima, J. Polym. Sci., B 35, 507 (1997).

48. J. Dong, Y. Ozaki, and K. Nakashima, Macromolecules 30, 1111 (1997).

49. O. N. Tretinnikov and K. Ohta, Macromolecules 35, 7343 (2002).

50. W. M. de Vos, P. M. Biesheuvel, A. de Keizer, J. M. Kleijn, M. A. Cohen Stuart Langmuir 24, 6575 (2008).

51. M. Jackson and H. Mantsch, Crit. Rev. Biochem. Mol. Biol. 30, 95 (1995).

52. C.J. Jung, Mol. Recognit. 13, 325 (2000).

53. R. Schweitzer-Stenner, Vibr. Spectrosc. 42, 98 (2006). 
Fig. 1. Ellipsometry spectra, obtained at an angle-of-incidence of $72^{\circ}$, for PAA- $b$-PMMA films on $\mathrm{Si}$ substrates before and after the adsorption of bovine submaxillary mucin at $\mathrm{pH}$ values of 3 and 10 , as indicated.

Fig. 2. Re $D$ spectra obtained from IR ellipsometry in the $1800-1000 \mathrm{~cm}^{-1}$ region for (a) the mucin layers on $\mathrm{Au}$ at a $\mathrm{pH}$ of 3 (solid line) and 7 (dashed line), (b) the PAA-b-PMMA copolymer film on $\mathrm{Au}$, and (c) the mucin/PAA-b-PMMA bilayers likewise at two pH values.

594 Curve 1 shows the experimental data, whereas curve 2 represents the sum of the spectra of the mucin layer and PAA-b-PMMA copolymer film. The vertical lines show the division of the spectra into four regions that will be considered individually. Arrows indicate trends in the intensity changes of $\mathrm{pH}$-sensitive bands in the mucin spectra.

Fig. 3. Re $D$ spectra in the carbonyl stretching region of the mucin/PAA- $b$-PMMA bilayers on $\mathrm{Au}$ at a $\mathrm{pH}$ of 3 (solid line) and 7(dashed line). The original spectra are in (a), and the results of their deconvolution are in (b). In both (a) and (b), curve 1 shows the experimental spectra and curve 2 represents the sum of the spectra of the mucin layer and the PAA-b-PMMA copolymer film.

Fig. 4. Proposed types of hydrogen bonding between the carboxyl groups of PAA- $b-$ PMMA copolymer and sialic acid residues of the mucin, illustrating (a) cyclic dimers and (b) face-on fashion bonding.

Fig. 5. Deconvolved Re $D$ spectra in the $1350-1000 \mathrm{~cm}^{-1}$ region of (a) the mucin layers on 608 and (c) the mucin/PAA- $b$-PMMA bilayers also at two different $\mathrm{pH}$ values. Curve 1 shows the 609 experimental data, whereas curve 2 represents the sum of the spectra of the mucin layer and the 610 PAA-b-PMMA copolymer film. Arrows indicate trends in the intensity changes of the bands in 611 the bilayer spectra caused by adhesion.

612 Fig. 6. A comparison of spectra obtained in the Amide I region. (a) IR absorption spectrum 613 obtained from a dry mucin powder, comparing (1) the original spectra and (2) the result of its 614 deconvolution. (b) Deconvolved Re D spectra obtained from (1) a mucin layer on Au and from 615 (2) mucin/PAA- $b$-PMMA bilayers, all at a $\mathrm{pH}$ of 3 and 7, as indicated.

616 Fig. 7. Deconvolved Re $D$ spectra in the Amide II and carboxylate stretching region (1600$6171350 \mathrm{~cm}^{-1}$ ) of (a) the mucin layers on $\mathrm{Au}$ at a $\mathrm{pH}$ of 3 (solid line) and 7 (dashed line), (b) the 618 PAA- $b$-PMMA copolymer film, and (c) the mucin/PAA-b-PMMA bilayers also at two 
619 different $\mathrm{pH}$ values. Curve 1 shows the experimental data, whereas curve 2 represents the sum 620 of the spectra of the mucin layer and the PAA- $b$-PMMA copolymer film.

621 
622 Table I. Adsorbed Mucin Layers’ Average

623 Thicknesses and Void Percentages Estimated

624 from Visible Ellipsometry Data Using the

625 Effective Medium Approximation.

\begin{tabular}{cccc}
\hline Polymer & pH & $\begin{array}{c}\text { Mucin layer } \\
\text { thickness } \\
(\mathrm{nm})\end{array}$ & $\begin{array}{c}\text { Voids } \\
(\text { vol. \%) }\end{array}$ \\
\hline PMMA & 7 & $3.9 \pm 0.8$ & $18.4 \pm 2.0$ \\
& 10 & $4.1 \pm 1.0$ & $17.3 \pm 1.6$ \\
& 3 & $4.5 \pm 1.2$ & $22.0 \pm 2.6$ \\
\hline PAA-b-PMMA & 7 & $8.1 \pm 1.0$ & $13.5 \pm 1.5$ \\
& & & \\
& 3 & $17.2 \pm 1.5$ & $9.0 \pm 1.0$ \\
\hline
\end{tabular}

626 

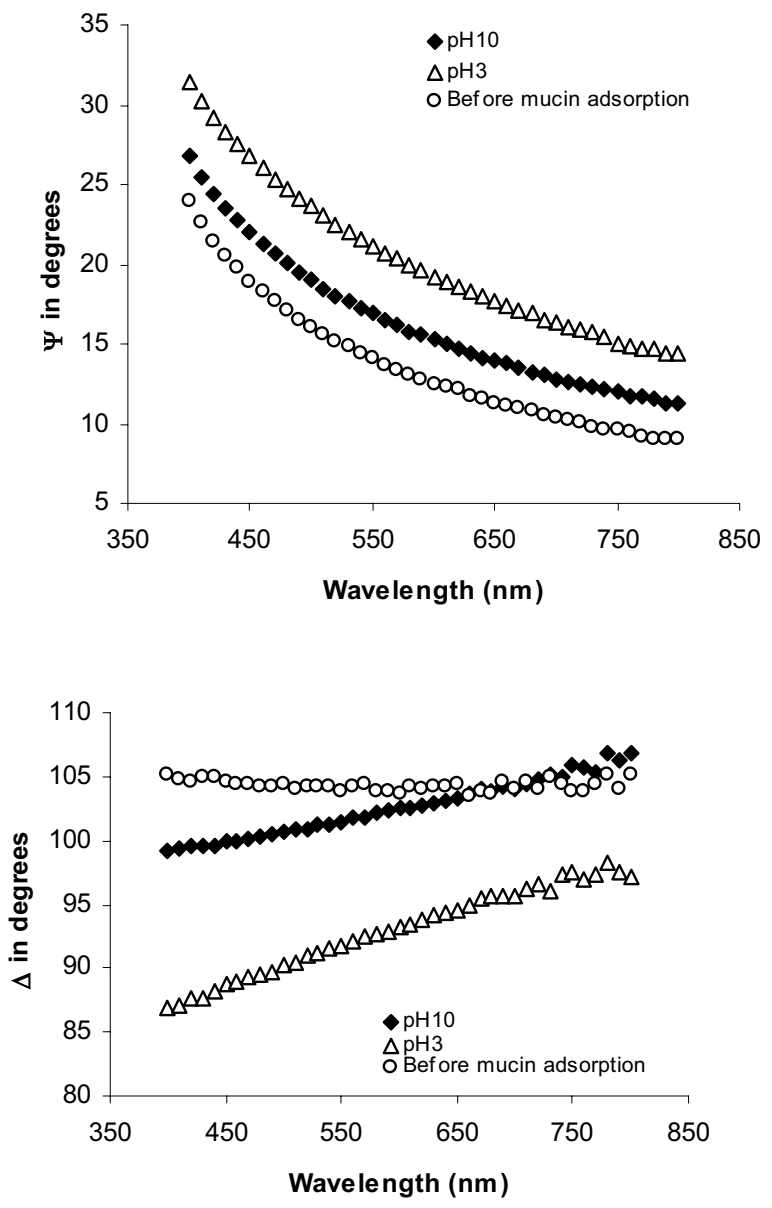

Figure 1 


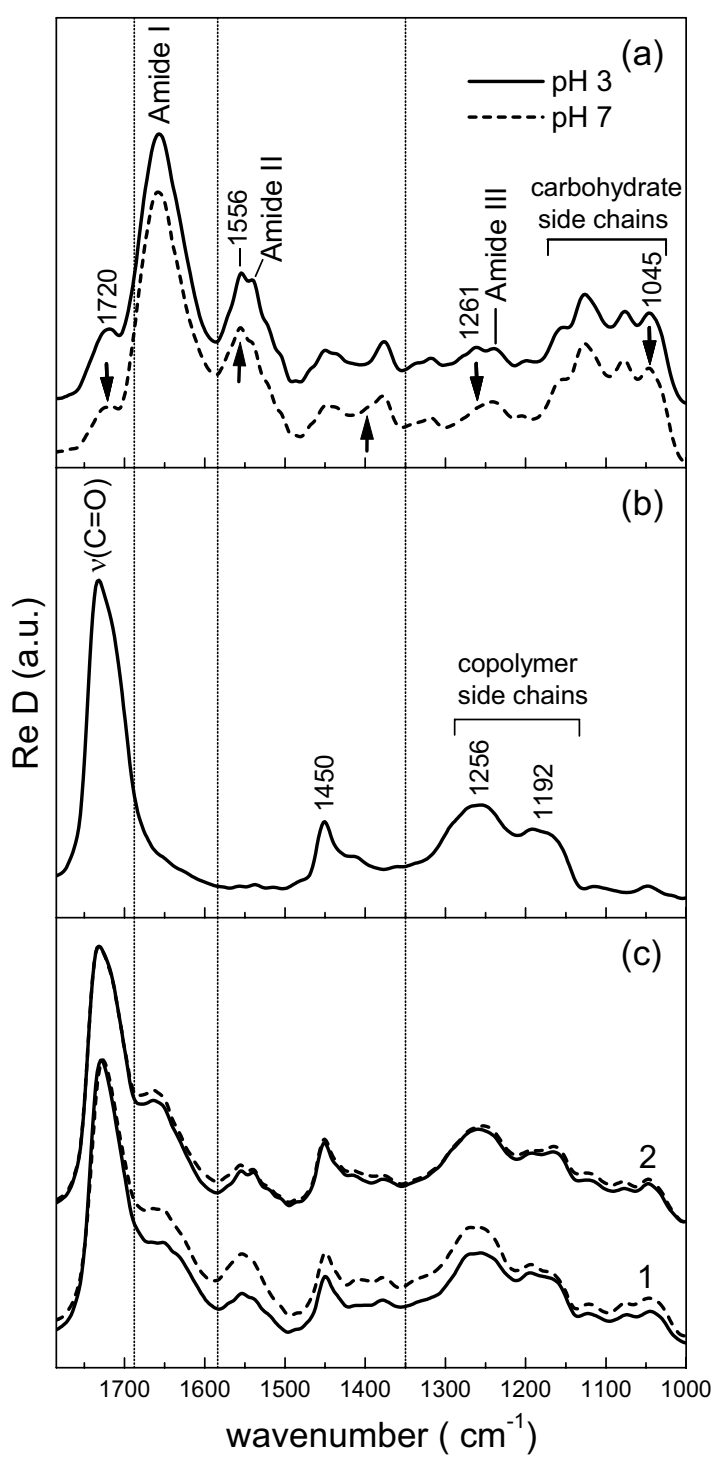

Figure 2 


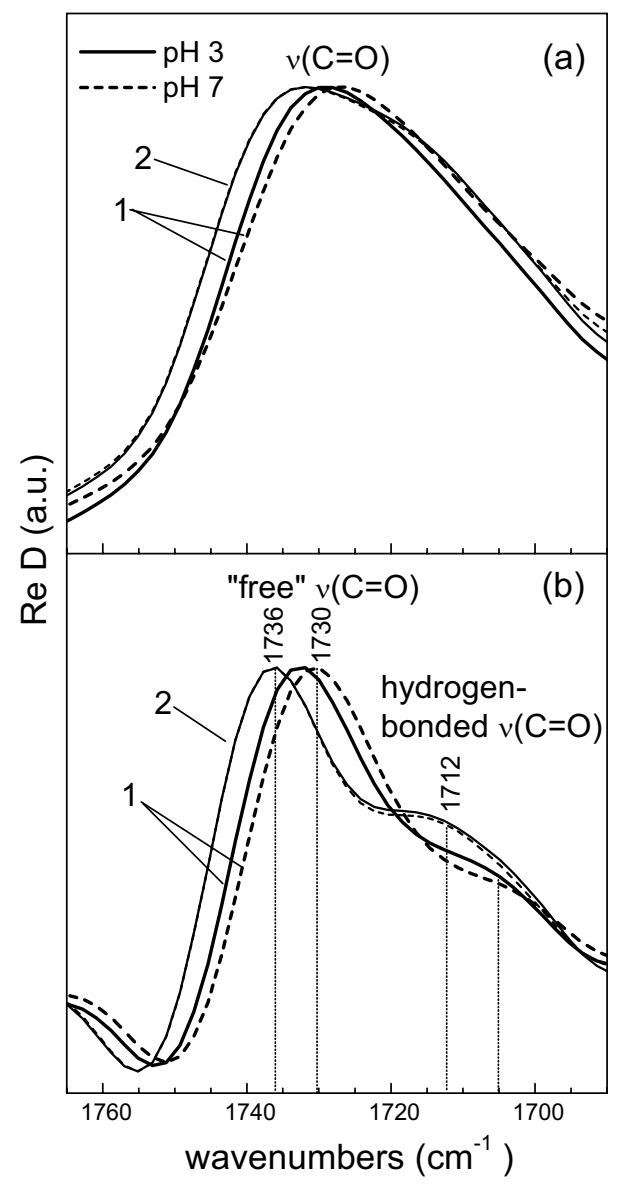

Figure 3 

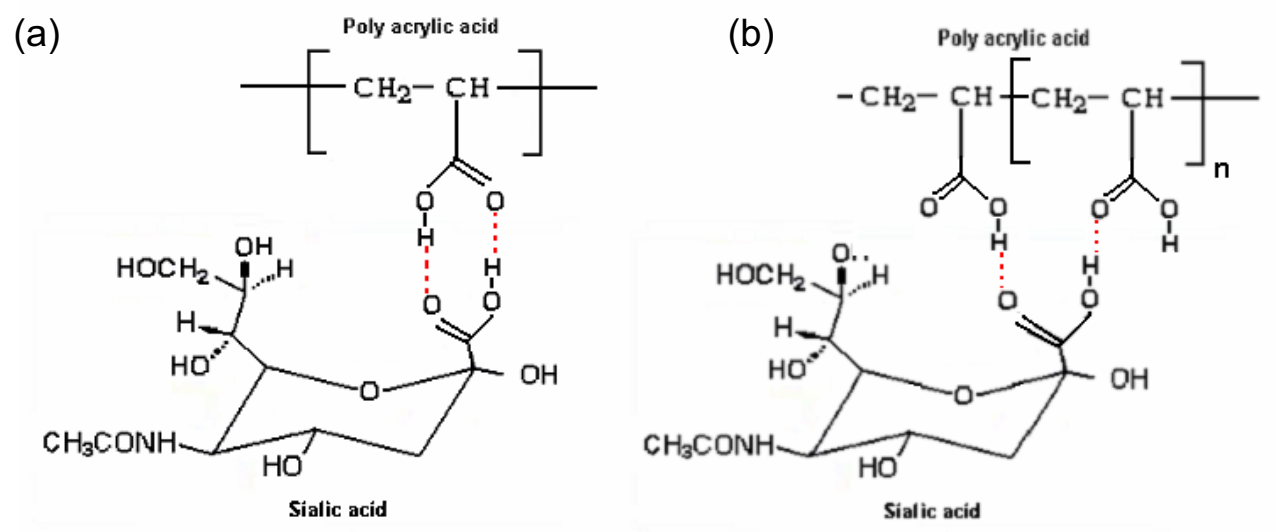

Figure 4 


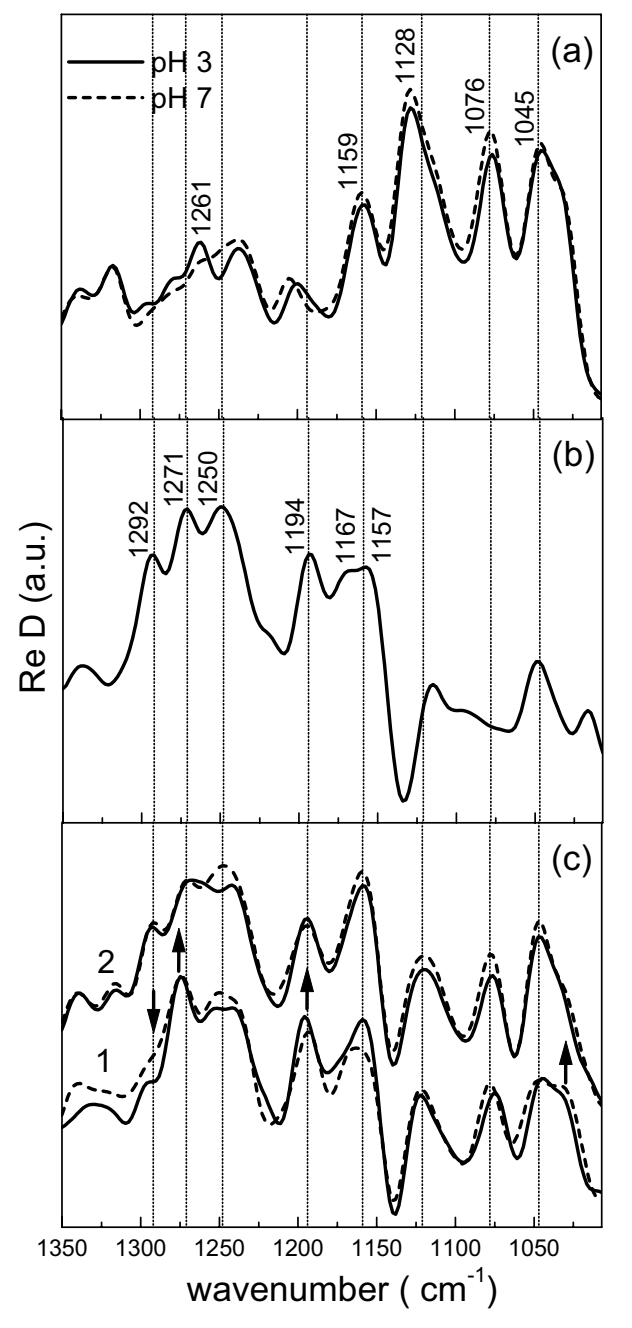

Figure 5 

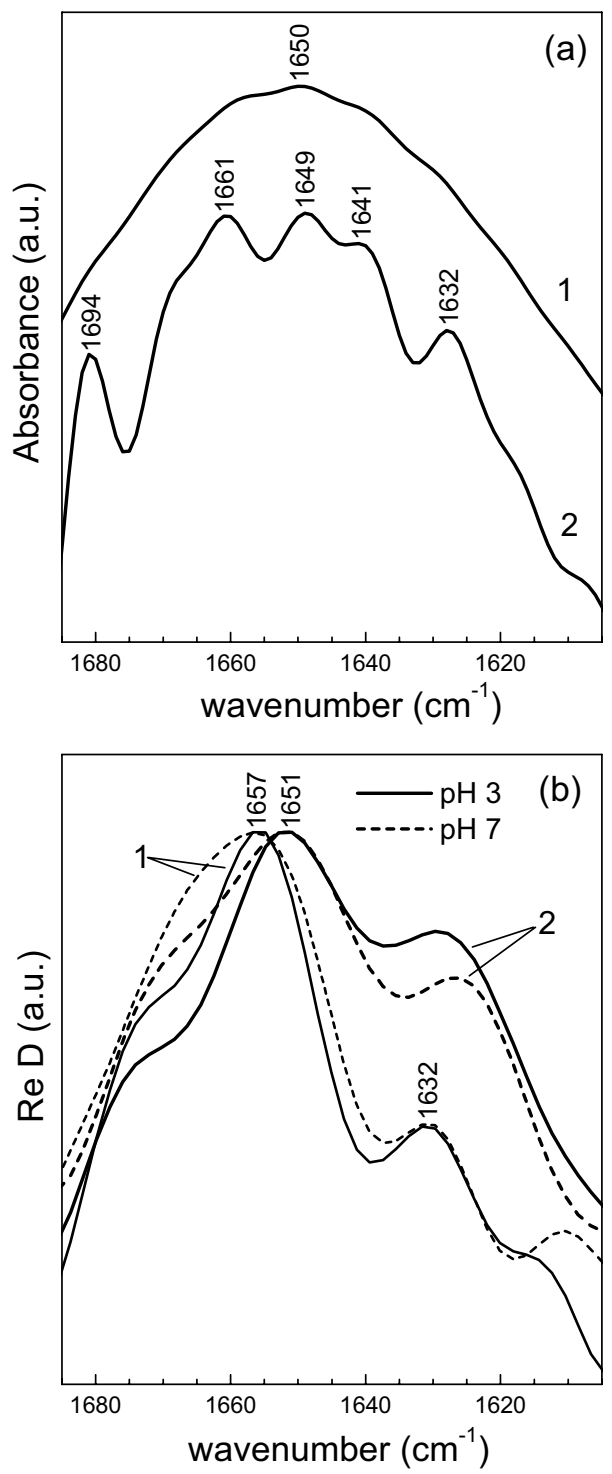

Figure 6 


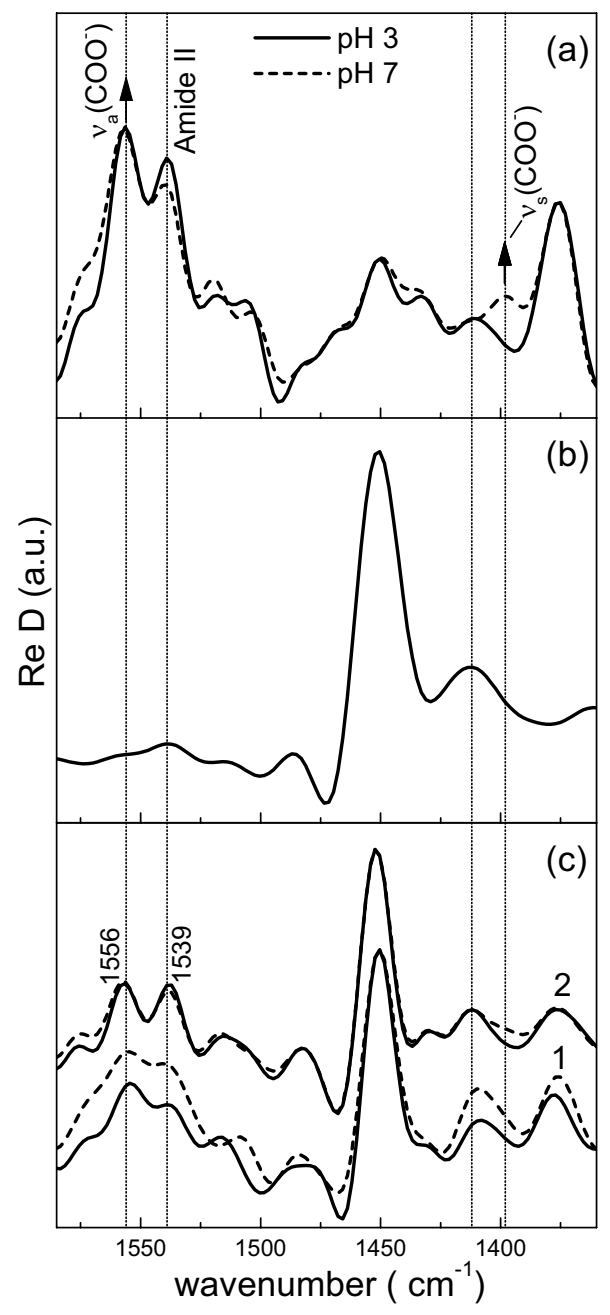

Figure 7 